\section{Brust- und Prostatakrebs: Vermehrte Schlafstörungen infolge der Tumortherapie?}

\author{
Viele Tumorpatienten leiden unter Schlafstörungen. Was im Einzelnen dazu \\ beiträgt, ist unklar. Stören z. B. adjuvante Therapien bei Brust- und Prostata- \\ krebs den Schlaf direkt oder indirekt über somatische Beschwerden?
}

\begin{abstract}
n der sekundären Analyse einer bevölkerungsbasierten Longitudinalstudie aus Kanada wurden Daten von 465 Patientinnen mit Brust- und 263 Patienten mit Prostatakrebs (Erstdiagnose, nicht metastasiert) ausgewertet. Sie beantworteten den ISI (Insomnia Severity Index) und einen Fragebogen zu somatischen Symptomen zu 6 verschiedenen Zeitpunkten: perioperativ (T1) sowie 2, 6, 10, 14 und 18 Monate danach (T2-T6). Separat untersucht wurden Effekte von Chemo-, Radio- und hormoneller Therapie. Dabei ist zu berücksichtigen, dass Brustkrebspatientinnen zwar über die gesamte Zeit eine Hormontherapie erhielten, aber nur bei T2 und T3 eine Chemooder Radiotherapie. Bei den Prostatakarzinompatienten konnte nur die Hormontherapie (T3-T6) ausgewertet werden, für Chemo- oder Radiotherapie waren die Zahlen zu niedrig $(\mathrm{n}<10)$.

Bei Patientinnen mit Brustkrebs waren Radiotherapie (insgesamt) und Chemotherapie (T2) signifikant mit zunehmenden Schlafstörungen (ISI) assoziiert, jedoch nicht die hormonelle Therapie. Bei Prostatakarzinompatienten ging der Androgenentzug dagegen durchweg mit erhöhten ISI-Werten einher. Dass sich für Brustkrebspatientinnen bezüglich Hormontherapie und Schlafstörungen kein signifikanter Zusammenhang zeigen ließ, geht möglicherweise auf einen CeilingEffekt zurück.

Bei Brustkrebspatientinnen wurden die Effekte von Chemo- und Radiotherapie auf den Schlaf signifikant durch Symptome vor allem vonseiten des Harntrakts (nächtlicher Harndrang), Übelkeit oder nächtliche Schweißausbrüche vermittelt. Letztere spielten als Auslöser der Schlafstörungen akut und längerfristig auch bei Prostatakrebspatienten eine große Rolle.
\end{abstract}

Fazit: Obwohl die Schlafstörungen bei Tumorerkrankungen höchstwahr- scheinlich multifaktoriell verursacht werden, sprechen die Ergebnisse dafür, dass Tumortherapien und von ihnen ausgelöste Nebenwirkungen zur Verschlechterung beitragen. Die gezielte Behandlung der Nebenwirkungen könnte helfen, Schlafstörungen bei diesen Patienten einzudämmen. Brigitte Schalhorn

Savard J et al. Cancer treatments and their side effects are associated with aggravation of insomnia: Results of a longitudinal study. Cancer. 2015;121(10):1703-11.

\section{Kommentar von Dr. Holzhauer:}

Schlaflosigkeit oder Insomnie ist ein bekanntes, relevantes und häufig anzutreffendes Leitsymptom bei Krebspatienten. Schlaflosigkeit im Kontext einer Tumorerkrankung ist multifaktoriell bedingt und wurde in zahlreichen Publikationen beschrieben.

In der vorgestellten Studie werden detailliert Nebenwirkungsspektren verschiedener adjuvanter, systemischer und lokaler Therapieformen bei Populationen von Brustkrebspatientinnen und Patienten mit Prostatakarzinom als potenzielle Trigger von Schlaflosigkeit in den Fokus gestellt. Interessant ist der sehr lange Beobachtungszeitraum mit Untersuchungen zur Symptomatik in der perioperativen Phase, nach 2, 6, 10, 14 und 18 Monaten. Wie die Forscher selbst erwähnen, liegt darin aber auch eine Beschränkung der Aussagen. Denn es ist durchaus denkbar, dass z. B. eine Patientin mit Brustkrebs zum Messzeitpunkt des Leitsymptoms Insomnie keine therapiebedingte Symptomatik oder eine geringere oder nachlassende Symptomatik hatte, weil die verursachende Behandlungsform schon deutlich vor der Messung beendet war.

In der vorliegenden Studie wird dabei ausschließlich auf die Rolle somatischer Symptome (unerwünschte Wirkungen) Bezug genommen, die durch die verschiedenen adjuvanten Therapieformen wie Chemotherapie, Strahlentherapie und endokri- ne Therapie ausgelöst wurden und damit Schlaflosigkeit initiiert oder verstärkt hatten. Im Vordergrund stehen dabei Symptome wie Nachtschweiß, Kopfschmerzen oder Übelkeit als Trigger von Schlaflosigkeit. Die Insomnie kann auch medikamentös durch die begleitende supportive Therapie induziert werden.

Der multifaktorielle Aspekt, auch die Koinzidenz möglicherweise schon im Vorfeld bestehender oder durch das Trauma der Diagnose verstärkter psychologischer, metabolischer oder auch immunologischer Alterationen wird zwar kurz erwähnt, nimmt aber dann leider keinen Raum mehr ein. Schlaflosigkeit wird ja auch als eine Facette im multifaktoriellen Spektrum des FatigueSymptomenkomplexes gesehen. Um hier die richtigen diagnostischen und dann auch therapeutischen Schlüsse und Behandlungskonsequenzen zu ziehen, ist es wünschenswert, dass auch weitere Komorbiditäten in die Gesamtbetrachtung des Aspektes Schlaflosigkeit einbezogen werden. In einer Studie aus dem Jahr 2014, die in diesem Zusammenhang interessant ist [Hall DL et al. Support Care Cancer. 2014;22(9):248995], wird die Angst vor einem Rezidiv ("cancer related uncertainty") als ein wichtiger Risikofaktor für das Auftreten von Schlaflosigkeit beschrieben.

\section{"Es sollten nicht nur somatische} Aspekte in die Gesamtbetrachtung von Insomnie einbezogen werden."

Genau wie ähnliche Publikationen zur Thematik von Fatigue und Nebenwirkungsassessment und in der Folge auch der Einsatz eines effektiven Nebenwirkungsmanagements ist die vorgestellte Studie von großer Bedeutung für die Supportivmedizin und die betreuten Patienten/innen. Denn im klinischen Alltag stehen die Wahrnehmung von und eine effektive Reaktion auf unerwünschte Nebeneffekte/Symptome, seien sie Therapie- oder Krankheitsfolge, häufig erst in der zweiten Reihe.

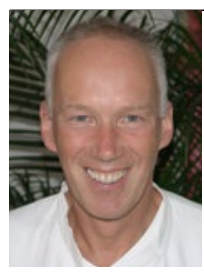

Dr. med. Peter Holzhauer Klinik Bad-Trissl, Oberaudorf Peter.Holzhauer@ klinik-bad-trissl.de 